\title{
QUAND BRANDYS NOUS IMAGINAIT LA POLOGNE
}

\author{
Jean-Yves POTEL (Mémorial de la Shoah, Paris)
}

J'ai lu Kazimierz Brandys à mesure que ses livres paraissaient en traduction française. Je conserve le souvenir de textes écrits au fil de la plume, avec de nombreuses digressions et des souvenirs, qui nous racontaient une Pologne à la fois fascinante, séduisante et profondément exaspérante. Cela tient sans doute à la manière dont il nous parlait... nous imaginait la Pologne. Il nous en a fait un roman, comme il en faisait un de sa vie. Son regard sur l'histoire a orienté ma connaissance de ce pays, de sa personnalité si insaisissable.

Un souvenir personnel d'abord. Lorsque je suis rentré de Gdańsk, en septembre 1980, j'étais ébloui par les images de la grève d'août. J'étais resté une semaine avec les grévistes dans le chantier naval occupé, j’y avais rencontré des gens attachants, je revenais la valise remplie de papiers et d'enregistrements, et la tête pleine de questions. Je voulais en faire un livre. Non pas un reportage ou un simple récit des événements. Une approche globale d'un événement historique. Je venais de lire Georges Duby, le grand historien médiéviste de l'école des Annales, qui révolutionnait justement la pensée de l'événement longtemps boudé par cette école. J'en avais retenu que l'on pouvait raconter une bataille - pour moi ce serait une grève - tout en traitant la profondeur historique et culturelle d'une époque ${ }^{1}$. Et quand j'ai parlé de mon projet à une amie polonaise, une vieille dame - ancienne résistante en France - , qui me traduisait mes documents et m'apprenait (un peu) le polonais, elle m'offrit un livre. "Tiens, lis-ça, tu comprendras mieux ». C'était un livre de Kazimierz Brandys paru récemment sous le titre En Pologne, c'est-à-dire nulle part ${ }^{2}$. J'ai froncé les sourcils. Je n'aimais guère l'usage que l'on faisait à tout bout de champ de cette citation d'Alfred Jarry. Pas un journaliste qui écrivait son premier article sur la Pologne sans qu'on y trouve cette expression. Mon amie m'apprit alors que c'était le titre français. Qu'en polonais il s'intitulait Irréalité. Voilà qui répondait mieux à mon projet. La lecture de

\footnotetext{
${ }^{1}$ Ce sera Scènes de grèves en Pologne, d'abord paru chez Stock en 1981, puis revu et augmenté chez Noir sur Blanc, en 2006.

${ }^{2}$ K. Brandys, Nierzeczywistość [Irréalité], Varsovie 1977, paru sous le titre En Pologne, c'est-à-dire nulle part, trad. fr. A. Lach, Paris 1978.
} 
ce livre fut une révélation. Il me donna des pistes pour suivre, à partir de mon expérience à Gdańsk, les traces de l'histoire et de l'imaginaire polonais.

En fait, Brandys m'a accompagné tout au long des années 1980 et 1990, je lisais immédiatement chacune de ses publications, je trouvais de vieux numéros des Temps modernes chez des bouquinistes ${ }^{3}$. Il fut sans doute l'écrivain polonais alors vivant, auquel j'étais le plus fidèle pendant ces années. Mon seul regret fut certainement de ne l'avoir jamais rencontré alors que je passais souvent près de chez lui, que je l'apercevais parfois, et que j'ai même écrit sur ses livres dans La Quinzaine littéraire.

Encore que. À chaque livre, j'avais l'impression de poursuivre une conversation avec une vieille connaissance. J'y trouvais un regard acéré sur son pays et, il faut l'ajouter tout de suite, sur le mien. Outre les Carnets rédigés pendant ses séjours en France, deux livres au moins m'ont ravi à ce propos. Je pense à Hôtel d'Alsace et autres adresses ${ }^{4}$ composé autour du portraits de trois écrivains - Oscar Wilde, André Gide et Paul Léautaud - et leurs mise en littérature de leurs propres vies. En confrontant les œuvres publiées à d'autres écrits comme les lettres d'Oscar Wilde, le Journal particulier longtemps inédit de Paul Léautaud, sur ses amours avec Mary Dormoy, ou le journal de la gouvernante de Gide, il s'amuse à révéler l'ambigüité de ces personnages, le « conflit entre le corps et l'âme, entre l'âme et les idées ». C'est un délice!

Plus étrange encore est son approche de l'exil et de la relation franco-polonaise à travers les aventures réelles ou fantasmagoriques d'un roi que les officiels français aiment à citer dans les discours — ce qui agace généralement les Polonais — et qu'il appelle Le Troisième Henri ${ }^{5}$. C'est le titre d'une pièce de théâtre rédigée par le personnage central de son roman, où l'on voit Henri de Valois, troisième fils de Catherine Médicis, justifier dans un long monologue post mortem son départ de la Pologne qui l'a pourtant élu roi. Brandys a, bien sûr, tout inventé. Il nous décrit la mise en scène et analyse les critiques. «J'aurais pu continuer d'être roi en Pologne », confie cet Henri III imaginaire — pour qui la Pologne «c'était des vacances » —, " mais j'aurais alors été étranger à ma propre biographie ». Vision étrange, fondamentale chez Brandys exilé, qu'illustre aussi la vie de l'auteur de cette pièce. Professeur de littérature polonaise dans l'État du Minnesota, il se prend à inventer des auteurs parce qu'il éprouve "une forte aversion » pour son cours sur Mikołaj Rej. Il «conçut douze silhouettes d'écrivains polonais du $\mathrm{XV}^{\mathrm{e}}$ au $\mathrm{XX}^{\mathrm{e}}$ siècle dont il imagina les œuvres ». Une façon écrit-il de "changer l'histoire en fiction ». N'est-ce pas ainsi que nous imaginons chacun notre pays ? Et pour occulter quoi ?

Ces souvenirs de lectures me conduisent à mon sujet. Je me suis rendu compte que cet accompagnement littéraire de ma découverte de la Pologne au début des années 1980, alors que j'avalais de gros livres d'histoire et de réflexions politiques pour rédiger ma thèse, cet accompagnement influença considérablement ma perception de ce pays imaginé. Brandys nous transmettait une Pologne complexe, complexée et attachante par ses extravagances, sinon ses aventures perdues. Il m'a en fait aidé

\footnotetext{
${ }^{3}$ Notamment le nr 159-160 où l'on peut lire une nouvelle Une visite inattendue (1959), et les deux livres dans la collection TM chez Julliard: La mère des rois, suivi de La défense de " Grenade », trad. Fr. V. Achères, Paris 1958 ; Lettres à Madame Z., trad. fr. A. Posner, Paris 1961.

${ }^{4}$ K. Brandys, Charaktery i pisma, Londres 1991, paru sous le titre Hôtel d'Alsace et autres adresses, trad. fr. J-Y. Erhel, Paris 1992.

${ }^{5}$ K. Brandys, Pomyst [L'idée], Varsovie 1974 ; paru sous le titre Le Troisième Henri, trad. fr. J.-Y. Erhel, Paris 1995.
} 
à comprendre les racines profondes de cette double vie si frappante, si troublante pour un observateur occidental de cette époque. Au début de En Pologne, c'est-à-dire nulle part il nous fournit un portrait de son père dans les années vingt et trente. Le narrateur - une sorte d'incarnation du destin de la Pologne au $\mathrm{XX}^{\mathrm{e}}$ siècle, un peu plus vieux que l'auteur - se souvient de son père rentrant à la maison le soir de l'assassinat du président Narutowicz en $1922^{6}:$ : J'avais huit ans [...] et il a dit 'ce sont eux'»", sans autre commentaire. Quelques années plus tard, le jour de son baccalauréat, il prend un méchant coup de canne sur la tête parce qu'il porte secours à un étudiant juif qui se fait rosser par un jeune nationaliste. Il se retrouve à l'hôpital. Et son père, rassuré sur son état de santé et sa réussite à l'examen, clôt l'incident. «Lorsque j'ai déclaré que dans les facultés polonaises on massacrait des gens pour la seule raison qu'ils étaient juifs, j'ai senti que mes paroles [...] provoquaient chez mon père une révolte d'un autre genre. De toute évidence, il m'en voulait d'appeler par leur nom des événements qu'il convenait de taire $»^{8}$. Le jeune homme est en rage. Il sait que son père connaît parfaitement la réalité. Le lendemain, il demande à son père occupé à manger des croissants, « s'il avait pris le même plaisir à déguster son croissant après l'assassinat de Narutowicz. A cet instant, mon père s'est brusquement levé et m'a giflé »" Cet homme écrit-il, « considérait toute allusion à l'antisémitisme et à la violence physique comme un vulgaire manque de tact. Et pourtant il n'ignorait pas ce qui se passait dans les facultés $»^{10}$. Cette «limitation» était «ancrée en lui ». Une «façon de passer sous silence la part de laideur que comporte la vérité. Ce refus d'observer avec acuité les faits et les hommes - comme une peur devant la réalité. Tout cela enfermé dans le moule de la nation $»^{11}$. Il vivait «dans l'irréalité polonaise, dans la sphère du mythe national », et «non à l'échelle de l'individu et de la civilisation. C'est sans doute pourquoi il se sentait plus libre que moi $»^{12}$.

Tout au long de son œuvre, Brandys revient sur cette manière d'être, et ce «moule de la nation ». Il les repère dans des situations très diverses. Il en fait même un grand jeu avec l'amour et la mort dans $R o n d o^{13}$, un roman vertigineux et magnifique sur la guerre, la clandestinité, la fiction et les réalités. Et surtout dans ses Carnets ${ }^{14}$. Je n'ai eu le temps de relire pour notre rencontre les trois volumes en français. J'en garde le souvenir d'un regard et de réflexions que l'on gagnerait à méditer aujourd'hui, alors qu'un certain nationalisme a repris des forces en Pologne. Brandys aborde constamment les deux faces d'un même événement, une Pologne visible, affichée, fière, et une Pologne à l'envers qu'il perçoit intimement, qu'il nous fait passer à travers des souvenirs, des digressions inattendues. Il décrypte, déconstruit en utilisant des outils tels l'ironie, l'humour, le chagrin, la colère ou l'admiration.

\footnotetext{
${ }^{6}$ Premier président élu dans la Pologne indépendante, proche de Piłsudski, assassiné deux jours après son élection par un fanatique de la droite nationaliste antisémite.

${ }_{8}^{7}$ K. Brandys, La Pologne..., p. 21.

${ }^{8}$ Ibid.

${ }^{9}$ Ibid.

${ }^{10}$ Ibid.

${ }^{11}$ Ibid., p. 27

${ }^{12}$ Ibid.

${ }^{13}$ K. Brandys, Rondo, Varsovie 1982; paru sous le titre Rondo, trad. fr. J.-Y. Erhel, Paris 1989.

${ }^{14}$ K. Brandys, trad. fr. Th. Douchy : Carnets de Varsovie 1978-1981, Paris 1985 ; Carnets Paris-New York-Paris 1982-1984, 1987 ; Carnets Paris 1985-1987, Paris 1990.
} 
Il décrit par exemple une réunion du Pen Club à Varsovie, le 11 novembre 1978, jour du soixantième anniversaire de l'indépendance de la Pologne. Il connaît tout le monde, «sont réunies plusieurs générations et des biographies très diverses », dans un «but commun» celui «d'exprimer leur désir de voir la Pologne indépendante et célébrer sa mémoire ${ }^{15}$. Ils sont " avides d'entendre une légende réconfortante ». Et voilà qu'un des orateurs a évoqué un État polonais entre les deux guerres «ni idéal ni tout à fait juste », et ce fut un « silence de mort. Le public était blessé » raconte-il. « On protestait contre le mépris des sentiments patriotiques». Et il se demande comment c'était possible. Tous ces gens, "l'élite de l'intelligentsia polonaise », la plupart était ses amis, tous condamnaient «l'auto-adulation nationale», connaissaient par cœur Gombrowicz, et voilà, « il a suffi de donner la parole à quelqu'un qui parlait en homme libre s'adressant à des hommes libres, pour que cette crème des intellectuels réagisse par un refus viscéral et que trois cent personnes crient au scandale $»^{16}$. "Pourquoi me demandais-je, puisqu'il avait dit la vérité sur les années d'avant-guerre ? Ce qu'il y a de pénible c'est de vivre dans une société où la vérité peut paraître incongrue $»^{17}$. Et plus loin : «Que faire contre ce besoin de mythe si profondément ancré dans des esprits par ailleurs évolués? $»^{18}$.

D'où sa réflexion permanente sur les responsabilités de l'intelligentsia tout au long de ces années, depuis les grèves de la faim, la fondation du KOR puis de Solidarność, puis «l'état de guerre», l'exil, etc. Ses Carnets sont d'ailleurs une des plus belles chroniques des interrogations et des émotions de ces années 1980 en Pologne.

Non sans retours réguliers sur l'expérience de la seconde guerre mondiale qui fut à l'évidence une période capitale de sa vie. Elle revient sans cesse dans ses livres. Que ce soit pour évoquer les conditions de vie sous l'occupation, des personnages disparus - beaucoup de portraits extraordinaires comme cette jeune femme Nina Willman avec qui il doit se cacher dans une maison de passe et qui servira de modèle à son amoureuse dans Rondo $o^{19}$, histoire qu'il cite plusieurs fois. L'antisémitisme bien sûr, ce qu'il entend quand le ghetto brûle et à maintes autres occasions — «j'étais alors un passant qui n'avait pas l'air d'un Juif, on parlait devant moi sans se gêner $»^{20}-$ ou ses remarques sur sa première vision du film de Claude Lanzmann, « une œuvre d'un genre nouveau et d'une importance capitale : le premier grand document filmé sur le comportement des hommes envers une communauté condamnée à mort ». Et à propos des paysans polonais aux propos antisémites, il les trouve tout simplement médiocres. "Ils sont simples, banals, communs $»^{21}$.

Il revient également à plusieurs reprises sur l'insurrection de Varsovie en 1944, sur l'opportunité de la décision, les responsabilités. Mais surtout, alors que Solidarność est menacé, fin mars 1981, sur la nécessité ou non d'un affrontement. On s'était préparé dans une «atmosphère fantastique » à la grève générale. Elle a été annulée au dernier moment par Wałęsa, au grand dam des militants. «Quand je dis : c'est une chance que la grève n'ait pas eu lieu, écrit-il, je bute contre le silence. La jeune femme avec qui je

\footnotetext{
${ }^{15}$ K. Brandys, Carnets de Varsovie 1978-1981, p. 25.

${ }^{16}$ Ibid.

${ }^{17}$ Ibid., p. 30 .

${ }^{18}$ Ibid.

${ }^{19}$ K. Brandys, Zapamiętane, Cracovie 1995, paru sous le titre De mémoire, trad. fr. J.-Y. Erhel, Paris 2003, p. 118.

${ }^{20}$ K. Brandys, Carnets de Varsovie 1978-1981, p. 91.

${ }^{21}$ K. Brandys, Carnets Paris 1985-1987, p. 105.
} 
cause n'avait pas vécu l'insurrection de Varsovie. En revanche, moi je me rappelle les journées d'avant l'explosion, quand les appartements se transformaient en forteresses. Alors aussi l'atmosphère était fantastique $»^{22}$.

Je pourrais encore citer de nombreux thèmes de ces Carnets écrits sur le ton de la conversation. Ainsi « du rôle du miracle dans la culture polonaise » qui produit une forme de narration si peu construite dans la littérature, mais surtout une certaine relation à l'événement à venir. Dans ses Carnets 1978-1981, il ironise souvent sur ce besoin de "résurrection". "Dieu lui-même semble nous encourager. Comme en réponse au slogan du parti, 'le Polonais réussit', il montre du doigt Wadowice. Devenir pape ? Un Polonais le réussit $»^{23}$. Voyez à ce propos, sa description des rues de Varsovie le jour de l'élection de Jean-Paul II : «Les gens couraient dans la ville en criant de joie. Un vieillard en larmes s'est jeté dans le bras de M. : Vous avez entendu Madame ? Miracle! Miracle !", et il commente: «Ce ne sont pas les poètes romantiques qui ont inventé le messianisme polonais... $»^{24}$. Il raille ceux qui rejettent sur le pape leur «attente nationale, inaccomplie et déçue. Toute leur polonité ils la confient à un Polonais, ils la lui mettent sur la tête avec ses épines $»^{25}$. Et ils l'implorent : «Sauve-nous, rends nous la dignité d'une grande nation et dis au monde qui nous sommes! Elle est poignante, la voix des Polonais qui s'élève vers le pape ${ }^{26}$.

Ce qui n'empêchera pas Brandys d'être gagné par la puissance et l'atmosphère de la foule qui accueille Jean-Paul II à Varsovie en juin 1979, la foule qui entendra son fameux «N'ayez pas peur». Il y voit un moment unique, une rupture justement avec cette irréalité souvent déplorée, un « événement vrai » qui préfigure août 1980. Il écrit après les accords de Gdańsk et la naissance de Solidarność : «Du jour au lendemain, nous nous sommes réveillés au milieu d'une société différente $»^{27}$.

Né en 1916 Brandys est mort à Paris, en 2000. C'était un écrivain lucide. Il nous offrait de belles réflexions sur l'histoire en devenir. Il pouvait être féroce. Il ne s'épargnait pas non plus. En le relisant aujourd'hui, je me rends compte combien il aimait son pays, sa culture. Et comment il savait nous en transmettre les vérités. Y compris les pires.

\footnotetext{
${ }^{22}$ K. Brandys, Carnets de Varsovie 1978-1981, p. 222.

${ }^{23}$ Ibid., p. 34.

${ }^{24}$ Ibid., p.88.

${ }^{25}$ Ibid., p.89.

${ }^{26}$ Ibid.

${ }^{27}$ Ibid., p. 105.
} 